Stanford-ITP-838

December 1987

Revised May 1992

\title{
One-Loop Threshold Effects in String Unification ${ }^{\star}$
}

\author{
VADIM S. KAPLUNOVSKY ${ }^{\dagger}$ \\ Department of Physics \\ Stanford University \\ Stanford, CA 94305
}

\begin{abstract}
Like grand unification of old, string unification predicts simple tree-level relations between the couplings of all unbroken gauge groups such as $S U(3)_{C}$ or $S U(2)_{W}$. I show here how to compute one-loop corrections to these relations for any four-dimensional model based on a classical vacuum of the heterotic string. The result can be used to calculate both $\sin ^{2} \theta_{W}$ and $\Lambda_{\mathrm{QCD}}$ in terms of $\alpha_{\mathrm{QED}}$ and $M_{\text {Planck }}$.
\end{abstract}

Printed (with minor errors) in Nuclear Physics B307 (1988)

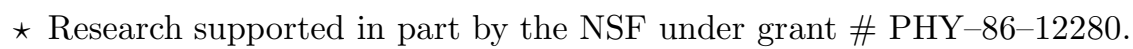

$\dagger$ Current (1992) address: Dept. of Physics, University of Texas, Austin, TX 78712. Current research is supported in part by the NSF under grant \#PHY-90-09850 and by the Robert A. Welch Foundation. 


\section{Introduction}

The term grand unification usually refers to unification of low-energy gauge interactions into a single gauge theory such as $S U(5), S O(10)$ or $E_{6}$ that is spontaneously broken at some high energy scale $M_{\mathrm{GUT}} \sim 10^{14}-10^{19} \mathrm{GeV}$. Not surprisingly, the main subjects of grand unified theories (GUTs) are the low-energy gauge couplings and the Georgi-Quinn-Weinberg (GQW) equations ${ }^{[1]}$ that relate these couplings to $M_{\mathrm{GUT}}$ and to each other. In the first approximation GQW equations can be written as

$$
\forall a: \quad \frac{16 \pi^{2}}{g_{a}^{2}(\mu)}=k_{a} \cdot \frac{16 \pi^{2}}{g_{\mathrm{GUT}}^{2}}+b_{a} \cdot \log \frac{M_{\mathrm{GUT}}^{2}}{\mu^{2}}+O(1)
$$

where index $a$ runs over low-energy gauge couplings and $\mu$ is some phenomenological scale such as $M_{W}$. Here the coefficients $k_{a}$ are the tree-level relations between the couplings (e.g. $k_{1}=5 / 3, k_{2}=k_{3}=1$ in $S U(5)^{[2]}$ ) while $b_{a}$ are the effects of couplings' renormalization below $M_{\mathrm{GUT}}$ : they are related to the one-loop $\beta$-functions via $\beta_{a}=b_{a} \cdot g_{a}^{3} / 16 \pi^{2}$. For a given grand unified model both $b_{a}$ and $k_{a}$ are fixed numbers and there are only two free parameters: $g_{\mathrm{GUT}}$ and $M_{\mathrm{GUT}}$. Hence, GQW equations yield one parameterless relation between the three phenomenologically known couplings $g_{1}, g_{2}$ and $g_{3}$ - or between related quantities $\alpha_{\mathrm{QED}}, \sin ^{2} \theta_{W}$ and $\log \Lambda_{\mathrm{QCD}}$; this relation is often called a prediction for $\sin ^{2} \theta_{W}$. In addition we can also compute $M_{\mathrm{GUT}}$ which is important for predicting the rate of proton decay and other processes that involve the unified theory.

The accuracy of equations (1) allows one to compute $\sin ^{2} \theta_{W}$ up to terms of order $O(\alpha)$ and $\log M_{\mathrm{GUT}}$ up to terms of order $O(1)$, which means that only the order of magnitude of $M_{\mathrm{GUT}}$ can be reliably predicted. In order to increase the accuracy of these predictions one has to solve the two-loop renormalization group equations for the couplings that are relevant below the GUT scale, and the boundary conditions for those equations should include one-loop corrections to the tree-level relations between the coupling. At the one-loop level of accuracy gauge 
couplings of the effective subthreshold-energy theory ${ }^{[3]}$ generally look like

$$
\forall a: \quad \frac{16 \pi^{2}}{g_{a}^{2}(\mu)}=k_{a} \cdot \frac{16 \pi^{2}}{g_{\mathrm{GUT}}^{2}}+b_{a} \cdot \log \frac{M_{\mathrm{GUT}}^{2}}{\mu^{2}}+\Delta_{a} .
$$

The new elements here are the explicit $O(1)$ threshold corrections $\Delta_{a}$ whose values depend on masses of heavy particles associated with the unification threshold.

String unification is more ambitious than ordinary grand unification: superstring theories ${ }^{[4]}$ are unified theories of all particle interactions. All currently known semi-realistic string models are based on the heterotic string theory, ${ }^{[5]}$ so this article deals exclusively with four-dimensional models of this kind. As far as low-energy gauge interactions are concerned, tree-level predictions of the string unification are similar to the GUT predictions: $\left(g_{a}^{\text {tree }}\right)^{-2}=k_{a} / g_{\text {string }}^{2}{ }^{[6]}$ so the GQW equations (1) apply equally well for both kinds of unification. Other tree-level couplings are also proportional to powers of $g_{\text {string }}$; in particular, the Newton's constant is given by $G_{N}=\alpha^{\prime} g_{\text {string }}^{2} / 8 \pi$. Moreover, $M_{\mathrm{GUT}}^{2}=O\left(1 / \alpha^{\prime}\right),{ }^{[7]}$ so $M_{\mathrm{GUT}} / g_{\text {string }}=O\left(M_{\text {Planck }}\right)$, which eliminates one free parameter from the GQW equations (1) and make both $\sin ^{2} \theta_{W}$ and $\Lambda_{\mathrm{QCD}}$ predictable in terms of $\alpha_{\mathrm{QED}}$ and $M_{\text {Planck }}$. Again, in order to make those predictions accurate one has to solve the two-loop renormalization group equations and to compute the threshold corrections $\Delta_{a}$ for the string threshold; this time, one also has to know the relation between $M_{\mathrm{GUT}}$ (as used in eqs. (2)) and $\alpha^{\prime}$ or $M_{\text {Planck }}{ }^{\star}$

The goal of this article is to derive a general formula for threshold effects in superstring theory. Specifically, I shall compute $\Delta_{a}$ for any four-dimensional model that is based on a classical vacuum of the heterotic string and has no tachyons; the model need not be space-time supersymmetric. This article is organized as follows: In the next section I derive a formula for threshold corrections in ordinary GUTs;

* Actually we only need to know the $O(1)$ term in $\log \left(\frac{M_{\mathrm{GUT}}}{M_{\mathrm{Planck}}}\right)$. Hence, we need not worry about string loops correcting the tree-level relation between $\alpha^{\prime}, g_{\text {string }}$ and $M_{\text {Planck }}$. For the same reason, an $O(1)$ term correction to $1 / g_{\text {string }}^{2}$ would also be unimportant. 
the result is not new, but the procedure provides a basis for the subsequent string calculation. The string calculation is presented in section 3; a general formula relating threshold effects in a string model to the model's massive spectrum concludes that section. In the final section $4 \mathrm{I}$ show how to apply the general formula to orbifold models and carry out the calculations for the $Z_{3}$ model.

\section{Thresholds in Ordinary Gauge Theories}

I would like to begin with an outline of a general procedure for calculating threshold effects. When we want to compute e.g. $\Delta_{\mathrm{QCD}}$ in some GUT, we first select a process that is dominated by QCD at energies below the unification scale - for example, gluon scattering. Second, we compute the one-loop amplitudes for this process as given by the two theories:

A) Complete unified theory that accounts for all particles, light and heavy.

$B)$ Effective low-energy theory, namely QCD with quarks and other light particles, but without any particles of mass $O\left(M_{\mathrm{GUT}}\right)$ or heavier.

At this point we do not use renormalization group techniques, but instead provide both theories with explicit ultraviolet regulators. The specifics of regularization schemes are not important as long as the ultraviolet regulators do not affect the infrared behavior of the respective theories; notice that this requirement excludes the dimensional regularization, but allows such schemes as Pauli-Villars, higher derivatives, proper-time cutoff, lattice, etc.

If the theory $B$ is the effective low-energy theory describing the same world as the more fundamental theory $A$, then the two amplitudes should agree with each other at low energies $E$, i.e.

$$
\mathcal{A}_{A}\left(g_{A}, \Lambda_{A}, E, \ldots\right)=\mathcal{A}_{B}\left(g_{B}, \Lambda_{B}, E, \ldots\right)+O\left(E^{2} / M_{\mathrm{GUT}}^{2}\right)
$$

(I assume both cutoffs $\Lambda_{A}$ and $\Lambda_{B}$ are chosen to be high enough to safely ignore any term proportional to a negative power of $\left.\Lambda_{A, B}\right)$. For given cutoffs $\Lambda_{A, B}$, equation 
(3) yields a relation between the two bare couplings $g_{A}$ and $g_{B}$. Having derived this relation, we can now use the renormalization group equations to replace the bare coupling $g_{B}$ with the running QCD coupling $g_{\mathrm{QCD}}(\mu)$; if the amplitude $\mathcal{A}_{B}$ was computed correctly, the cutoff $\Lambda_{B}$ should disappear from the resulting formula. Similarly, $g_{A}$ and $\Lambda_{B}$ can be replaced with the running unified coupling $g_{\mathrm{GUT}}\left(\mu^{\prime}\right)$. It remains to substitute $\mu=\mu^{\prime}=M_{\mathrm{GUT}}$, and we have a threshold relation between $g_{\mathrm{GUT}}$ and $g_{\mathrm{QCD}}$; other low-energy gauge couplings can be related to $g_{\mathrm{GUT}}$ in the same way. Note that the definition of a running coupling $g(\mu)$ depends on a choice of a renormalization scheme. Hence the values of the one-loop threshold corrections $\Delta_{a}$ depend on a choice of renormalization schemes for both $g_{a}$ and $g_{\mathrm{GUT}}$; for the gauge couplings, I shall use the $\overline{\mathrm{DR}}$ scheme (modified minimal subtraction for dimensional reduction) throughout this article.

The above procedure is general enough to be valid in any unified theory, be it an ordinary GUT or a superstring-based model. However, when it comes to choosing a process, the state-of-the-art string theory imposes a limitation: only on-shell amplitudes can be computed at present. Fortunately, an on-shell amplitude does not have to be a scattering amplitude: Lagrangian density of a non-trivial background that obeys equations of motions is also on-shell. ${ }^{\star}$ The simplest background for probing the gauge coupling $g_{a}$ is given by a uniform (i.e., covariantly constant) gauge field $F_{\mu \nu}^{a}$; the effective Lagrangian density of this background has been used before for comparing unlike ultraviolet regulators for QCD such as lattice versus Pauli-Villars, ${ }^{[8]}$ and I simply generalize this approach to the case when a unified theory acts as a QCD cutoff.

For any background that solves the classical equations of motion, quantum field theory gives the following expression for the one-loop contribution to its Lagrangian

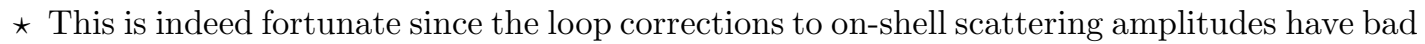
infrared problems in any interacting theory with massless particles. In string theory those problems become much worse because we can no longer separate one-particle irreducible Feynman diagrams from corrections to external legs.
} 
density:

$$
\mathcal{L}_{1}=\frac{1}{2} \operatorname{Str}(\log L) \equiv \int_{0}^{\infty} \frac{d t}{2 t} C_{\Lambda}(t) \cdot \operatorname{Str}\left(e^{-t L}\right)
$$

where the super-trace is taken over the Hilbert space of all quantum fields (including ghosts). Operator $L$ is the first-quantized Lagrangian: $L=M^{2}-D^{\lambda} D_{\lambda}$ for scalar and ghost fields, $L=M^{2}-D^{\lambda} D_{\lambda}-\frac{1}{2} F_{\mu \nu} \gamma_{\mu} \gamma_{\nu}$ for spinors ( $L$ is the second order Lagrangian) and $(L)_{\mu \nu}=M^{2}-D^{\lambda} D_{\lambda}-2 F_{\mu \nu}$ for vectors in the background Feynman gauge ( $D_{\mu}$ are covariant derivatives with respect to the background gauge potentials $A_{\mu}$ ). The integration variable $t$ is the proper time and $C_{\Lambda}(t)$ is an ultraviolet regulator.

The bare coupling $1 / g_{a}^{2}$ is the coefficient of the $\frac{1}{4} F_{\mu \nu}^{a}{ }^{2}$ in the bare Lagrangian, so what we need is the coefficient $\mathcal{W}_{a}$ of a similar term in $\mathcal{L}_{1}$. Let me therefore expand the exponential in (4) to the second order in $A_{\mu}$ and $F_{\mu \nu}$, substitute $F_{\mu \nu}(x)=$ const, $A_{\mu}(x)=\frac{-1}{2} F_{\mu \nu} x^{\nu}$ and do the $x$ integral (or the equivalent momentum integral) that is implicit in the super-trace. The result is

$$
\mathcal{W}_{a}^{\text {field }}=\frac{1}{16 \pi^{2}} \int_{0}^{\infty} \frac{d t}{t} C_{\Lambda}(t) \cdot\left[\mathbf{B}_{a}(t) \stackrel{\text { def }}{=} 2 \operatorname{str}\left(Q_{a}^{2}\left(\frac{1}{12}-\chi^{2}\right) e^{-t M^{2}}\right)\right]
$$

where the super-trace is now taken over the spectrum of physical particles of the theory, operator $Q_{a}$ is a generator of the gauge-group $a, \chi$ is the helicity operator and $M$ is the mass operator. Notice that the integral in (5) diverges in the infrared $t \rightarrow \infty$ limit; the divergence is logarithmic, with a coefficient related to the $\beta$ function of the low-energy theory:

$$
\mathbf{B}_{a}(t) \underset{t \rightarrow \infty}{\longrightarrow}-\frac{11}{3} \operatorname{tr}_{V}\left(Q_{a}^{2}\right)+\frac{2}{3} \operatorname{tr}_{F}\left(Q_{a}^{2}\right)+\frac{1}{3} \operatorname{tr}_{S}\left(Q_{a}^{2}\right) \equiv b_{a}
$$

where the traces are taken over the massless charged particles and count each CPT-conjugate particle-antiparticle pair only once. 
Formula (5) is applicable to any kind of a gauge theory. The effective lowenergy theory has the same massless spectrum as the complete unified theory but no massive particles. Hence, while for the complete theory $\mathbf{B}_{a}(t)$ is $t$-dependent, for the subthreshold theory, $\mathbf{B}_{a}^{\mathrm{eff}}(t) \equiv b_{a}$. For both theories $\left(g_{a}^{\text {bare }}\right)^{-2}+\mathcal{W}_{a}$ should have the same value, so

$$
\begin{aligned}
\frac{16 \pi^{2}}{g_{a}^{2}}-\frac{16 \pi^{2} k_{a}}{g_{\mathrm{GUT}}^{2}} & =\int_{0}^{\infty} \frac{d t}{t}\left(C_{\mathrm{GUT}}(t) \cdot \mathbf{B}_{a}(t)-C_{\mathrm{eff}}(t) \cdot b_{a}\right) \\
& =2 \operatorname{str}_{M \neq 0}\left(Q_{a}^{2}\left(\frac{1}{12}-\chi^{2}\right) \log \frac{\Lambda_{\mathrm{PV}}^{2}}{M^{2}}\right),
\end{aligned}
$$

where I have computed the integral for the case when the same Pauli-Villars regulator $C_{\mathrm{PV}}(t)=1-e^{-t \Lambda^{2}}$ is used for both theories. It remains to translate the bare couplings on the left hand side of (7) into the running $\overline{\mathrm{DR}}$ couplings evaluated at $\mu=M_{\mathrm{GUT}}$; the result is the following expression for the threshold corrections in grand unified models:

$$
\Delta_{a}=2 \operatorname{str}_{M=O\left(M_{\mathrm{GUT}}\right)}\left(Q_{a}^{2}\left(\frac{1}{12}-\chi^{2}\right) \log \frac{M_{\mathrm{GUT}}^{2}}{M^{2}}\right) .
$$

I would like to conclude this section with a remark that formula (8) applies not just to the GUT threshold, but to any threshold that separates two ordinary gauge theories. This includes any intermediate-energy thresholds a string-based model might possess: once we have an effective four-dimensional field theory at some sub-Planck energy scale, its further evolution towards lower energies can be handled with ordinary field-theoretical techniques. It is the string threshold itself that requires a different formula; I shall derive it in the next section. 


\section{One-Loop Effects at the String Threshold}

Having established a general procedure for computing one-loop threshold effects, let me now apply it to the string threshold. My starting point is the string analogue of formula (4):

$$
\mathcal{L}_{1}^{\text {string }}=\int_{\Gamma} \frac{d^{2} \tau}{\tau_{2}} \cdot\left[Z(\tau, \bar{\tau}) \stackrel{\text { def }}{=} \operatorname{Tr}\left(q^{H} \bar{q}^{\bar{H}}\right)\right],
$$

where $Z(\tau, \bar{\tau})$ is the partition function of the string, $H \stackrel{\text { def }}{=} L_{0}-\frac{c}{24}$ and $\bar{H} \stackrel{\text { def }}{=} \bar{L}_{0}-\frac{\bar{c}}{24}$

are the left-moving and the right-moving world-sheet Hamiltonians, $g \stackrel{\text { def }}{=} e^{2 \pi i \tau}$, and $\Gamma \stackrel{\text { def }}{=}\left\{\tau=\tau_{1}+i \tau_{2}: \tau_{2}>0,\left|\tau_{1}\right|<\frac{1}{2},|\tau|>1\right\}$ is the modular domain of the one-loop world sheet. Formula (9) was derived by Polchinski ${ }^{[9]}$ for the closed bosonic string in 26 flat space-time dimensions, or in any other background that obeys the classical equations of motion; such backgrounds correspond to conformally invariant field theories on the world sheet. For the heterotic string one should replace a single partition function $Z$ with a sum of separate partition functions for the four spin structures:

$$
Z(\tau, \bar{\tau}) \mapsto \frac{1}{2} \sum_{s_{1}, s_{2}}\left[Z_{s_{1} s_{2}}(\tau, \bar{\tau}) \stackrel{\text { def }}{=} \operatorname{Tr}_{s_{1}}\left((-)^{s_{2} \cdot F} q^{H} \bar{q}^{\bar{H}}\right)\right]
$$

where traces are now taken over distinct Ramond $\left(s_{1}=1\right)$ and Neveu-Schwarz $\left(s_{1}=0\right)$ sectors of the Hilbert space.

Let us chose a classical vacuum of the heterotic string that gives rise to a four-dimensional model with some massless particles such as gauge bosons. When some space-time fields $\phi$ acquire vacuum expectation values, this amounts to a perturbative change in the string's background. As long as a perturbed background remains a solution to classical equations of motion, formula (9) will remain applicable. The partition function $Z_{\phi}$ of the new background is perturbatively related 
to the original background via

$$
Z_{\phi}=Z_{\phi=0} \cdot\left(1+\int d^{2} \zeta\left\langle V_{\phi}(\zeta, \bar{\zeta})\right\rangle+\frac{1}{2} \iint d^{2} \zeta_{1} d^{2} \zeta_{2}\left\langle V_{\phi}\left(\zeta_{1}, \bar{\zeta}_{1}\right) V_{\phi}\left(\zeta_{2}, \bar{\zeta}_{2}\right)\right\rangle+\cdots\right)
$$

where $\zeta$ 's are coordinates on the world torus, $V_{\phi}(\zeta, \bar{\zeta})$ are string vertices corresponding to the background fields $\phi$, and $\langle V \cdots V\rangle$ are their correlation functions evaluated in the unperturbed vacuum. For the even spin structures all vertices $V_{\phi}$ should be taken in the zero-ghost-number picture; in the odd (Ramond-Ramond) spin structure one of the vertices should be taken in a different picture and an additional operator should be inserted in the correlation function. ${ }^{[10]}$ Fortunately, the only feature of the Ramond-Ramond one-loop amplitudes that is relevant in this article is that all such amplitudes contain the $\epsilon^{\alpha \beta \gamma \delta}$ tensor. The amplitude we would like to compute $-F_{\mu \nu}^{2}$ term in $\mathcal{L}_{1}$ - does not contain the $\epsilon$ tensor, so it will get no contribution from the Ramond-Ramond partition function $Z_{11}\left(F_{\mu \nu}\right)$.

Before we plug a perturbation that turns on a uniform gauge field and no other fields into (11), we have to verify that this perturbation obeys the classical string equations of motion. Perturbatively, those equations can be derived from an effective Lagrangian, ${ }^{[11]}$ and in the absence of charged background fields, Yang-Mills equations become $\delta \mathcal{L} / \delta A^{\mu}=D^{\nu}\left(\delta \mathcal{L} / \delta F^{\mu \nu}\right)=0$; such equations are always satisfied by covariantly constant $F_{\mu \nu}$ regardless of details of $\mathcal{L}\left(F_{\mu \nu}\right)$. On the other hand, non-zero $F_{\mu \nu}^{a}$ results in non-zero classical stress-energy tensor $T_{\mu \nu}=\left(F_{\mu \lambda}^{a} F_{\nu}^{a \lambda}-\right.$ $\left.\frac{1}{4} g_{\mu \nu} F_{\alpha \beta}^{a} F^{a \alpha \beta}\right) /\left(g_{a}^{\text {tree }}\right)^{2}+O\left(F^{4}\right)$. Therefore, Einstein equations can only be satisfied if we turn on the background gravitational field in addition to the gauge field, and both kind of vertices should appear in the perturbative expansion (11). Terms that are quadratic in $F_{\mu \nu}^{a}$ can be written as:

$$
\left.\frac{Z(F)}{Z(0)}\right|_{F^{2}}=\int d^{2} \zeta\left\langle V_{\text {grav }}(\zeta, \bar{\zeta})\right\rangle+\frac{1}{2} \iint d^{2} \zeta_{1} d^{2} \zeta_{2}\left\langle V_{\text {gauge }}\left(\zeta_{1}, \bar{\zeta}_{1}\right) V_{\text {gauge }}\left(\zeta_{2}, \bar{\zeta}_{2}\right)\right\rangle .
$$

Let me consider the second term first. For the background $F_{\mu \nu}=$ const, $A_{\mu}(X)=\frac{-1}{2} F_{\mu \nu} X^{\nu}$, a zero-ghost-number-picture vertex for the background gauge 
field $^{[12]}$ looks like

$$
V_{\text {gauge }}(\zeta, \bar{\zeta})=\frac{i \alpha^{\prime}}{4 \pi} F_{\mu \nu}\left(X^{\mu}(\zeta, \bar{\zeta}) \bar{\partial} X^{\nu}(\zeta, \bar{\zeta})+\Psi^{\mu}(\bar{\zeta}) \Psi^{\nu}(\bar{\zeta})\right) \cdot J^{a}(\zeta)
$$

where $X^{\mu}$ are space-time coordinates, $\Psi^{\mu}$ are their fermionic partners and $J^{a}$ is a current in the Kac-Moody algebra that generates the gauge group a under consideration. The correlation function of two such vertices can be written as

$$
\begin{aligned}
\left\langle V_{\text {gauge }}(\zeta, \bar{\zeta}) V_{\text {gauge }}(0)\right\rangle=\frac{\alpha^{\prime 2}}{8 \pi^{2}} F_{\mu \nu}^{2} \cdot\left\langle J^{a}(\zeta)\right. & \left.J^{a}(0)\right\rangle \times \\
& \times\left(\langle\Psi(\bar{\zeta}) \Psi(0)\rangle^{2}-\langle\bar{\partial} X(\zeta, \bar{\zeta}) X(0)\rangle^{2}\right)
\end{aligned}
$$

(making use of the translational symmetry of the torus, I have set $\zeta_{2}=0$ ). Now consider the Kac-Moody factor in (14). In a sector of the Hilbert space that has definite charge $Q_{a}$ with respect to the zero mode of $J^{a}$, one has

$$
\left\langle J^{a}(\zeta) J^{a}(0)\right\rangle(\tau)=\sum_{n} \frac{\pi^{2} k_{a}}{\sin ^{2}(\pi \zeta+n \pi \tau)}-4 \pi^{2} Q_{a}^{2}
$$

in a more general situation the $Q_{a}^{2}$ term should be averaged. All other terms are proportional to the Kac-Moody central charge $k_{a}$ and do not otherwise depend on the choice of a particular gauge group $a$. This is a consequence of the requirements that this correlation function should be holomorphic, single-valued on the torus, and have a $k_{a} / \zeta^{2}$ singularity for $\zeta \rightarrow 0$; these requirements completely determine $\left\langle J^{a}(\zeta) J^{a}(0)\right\rangle$ up to a constant with respect to $\zeta$. Note that denoting the KacMoody central charges with $k_{a}$ is not in collision with the earlier use of the same symbols in eq (2): it is these central charges that determine the ratios between the tree-level gauge couplings. ${ }^{[6]}$ for

Evaluation of the gravitational term in (12) requires an explicit expression for $V_{\text {grav }}$ that corresponds to a solution of the Einstein equations with the source $T_{\mu \nu}$. However, I do not have to solve those equations to notice that $V_{\text {grav }} \propto T_{\mu \nu} \propto$ $\left(g_{a}^{\text {tree }}\right)^{-2} \propto k_{a}$ and does not otherwise depend on the choice of the gauge group $a$. 
Therefore, at this point I can write the following formula for $\mathcal{W}_{a}^{\text {string. }}$

$$
\begin{aligned}
\mathcal{W}_{a}^{\text {string }}=k_{a} \cdot Y-\frac{1}{2} \alpha^{\prime 2} \int_{\Gamma} d^{2} \tau \sum_{\text {even } \mathbf{s}} & \operatorname{Tr}_{s_{1}}\left((-)^{s_{2} F} q^{H} \bar{q}^{\bar{H}} \cdot Q_{a}^{2}\right) \times \\
& \times \int d^{2} \zeta\left(\langle\Psi(\bar{\zeta}) \Psi(0)\rangle_{\mathbf{s}}^{2}-\langle\bar{\partial} X(\zeta, \bar{\zeta}) X(0)\rangle^{2}\right)
\end{aligned}
$$

where $Y$ stands for the total of $a$-independent terms. Notice that the $Q_{a}^{2}$ operator has been brought inside the trace - that is what I meant by averaging the $Q_{a}^{2}$ term.

The trace in (16) obviously depends on a particular string vacuum. Nevertheless, all four-dimensional vacua share some of the world-sheet degrees of freedom, namely the four $X^{\mu}, \Psi^{\mu}$ and the superconformal ghosts. Hence, we can decompose the trace in (16) as

$$
Z_{4 \mathrm{~d}+\mathrm{gh}}(\tau, \bar{\tau}, \mathbf{s}) \cdot \operatorname{Tr}_{s_{1}}\left(Q_{a}^{2} \cdot(-)^{s_{2} F} q^{H} \bar{q}^{\bar{H}}\right)_{\mathrm{int},}
$$

where the second factor involves the 'internal' degrees of freedom only. The first factor - the partition function of space-time and ghost degrees of freedom is common to all four-dimensional models. The effect of ghosts is to cancel the contribution of the non-zero modes of two of the four $X^{\mu}$ and two of $\Psi^{\mu}$; the remaining factor is a minus sign for all spin structures other than (NS,NS). The non-zero modes of the other two $X^{\mu}$ contribute $Z_{X}(\tau, \bar{\tau})=|\eta(\tau)|^{-4}$, and the contribution of two $\Psi^{\mu}$ can also be expressed in terms of $\eta$-functions:

$$
Z_{\Psi}(\bar{\tau}, \mathbf{s})=\frac{1}{\eta^{2}(\bar{\tau})} \cdot \begin{cases}\eta^{2}\left(\frac{\bar{\tau}+1}{2}\right) & \text { for } \mathbf{s}=(\mathrm{NS}, \mathrm{NS}) \\ \eta^{2}\left(\frac{\bar{\tau}}{2}\right) & \text { for } \mathbf{s}=(\mathrm{NS}, \mathrm{R}) \\ 2 \eta^{2}(2 \bar{\tau}) & \text { for } \mathbf{s}=(\mathrm{R}, \mathrm{NS})\end{cases}
$$

Finally, the contribution of zero modes of $X^{\mu}$ ( $\Psi^{\mu}$ have no zero modes for even spin structures) is simply

$$
Z_{0}(\tau, \bar{\tau})=\int \frac{d^{4} p}{(2 \pi)^{4}} \exp \left(-\pi \alpha^{\prime} \tau_{2} p^{2}\right)=(2 \pi)^{-4}\left(\alpha^{\prime} \tau_{2}\right)^{-2}
$$

The last factor in eq. (16) — the world sheet integral — is also common to all heterotic string models and can be computed explicitly. The details of this 
computation are rather boring, but the result can be summarized in terms of $Z_{\Psi}$ :

$$
\int \frac{d^{2} \zeta}{\tau_{2}}\left(\langle\Psi(\bar{\zeta}) \Psi(0)\rangle_{\mathbf{s}}^{2}-\langle\bar{\partial} X(\zeta, \bar{\zeta}) X(0)\rangle^{2}\right)=4 \pi i \frac{d}{d \bar{\tau}} \log Z_{\Psi}(\bar{\tau}, \mathbf{s}) .
$$

This completes the analysis of eq. (16), which can now be rewritten as

$$
\mathcal{W}_{a}^{\text {string }}=\frac{1}{16 \pi^{2}} \int_{\Gamma} \frac{d^{2} \tau}{\tau_{2}} \mathcal{B}_{a}(\tau, \bar{\tau})+k_{a} \cdot Y,
$$

where

$$
\mathcal{B}_{a}(\tau, \bar{\tau})=\frac{2}{|\eta(\tau)|^{4}} \cdot \sum_{\text {even } \mathbf{s}}(-)^{s_{1}+s_{2}} \frac{d Z_{\Psi}(\bar{\tau}, \mathbf{s})}{2 \pi i d \bar{\tau}} \cdot \operatorname{Tr}_{s_{1}}\left(Q_{a}^{2} \cdot(-)^{s_{2} F} q^{H} \bar{q}^{\bar{H}}\right)_{\mathrm{int}}
$$

The integral in eq. (21) is logarithmically divergent in the infrared $\tau_{2} \rightarrow \infty$ limit. This is similar to the divergence of the proper-time integral in eq. (5); in fact, both divergences have the same coefficient $b_{a}$. To see that, consider the limit of $\mathcal{B}_{a}\left(\tau_{1}, \tau_{2}\right)$ as $\tau_{2}$ becomes very large. Unless we are dealing with a string model that has charged tachyons - an obviously unrealistic case - the traces in eq. (22) are dominated in this limit by massless particles that have $H=\bar{H}=0$. Hamiltonians $H$ and $\bar{H}$ are totals of space-time, ghost and internal components; evaluating the space-time and ghost components, we find that massless vectors have $(H, \bar{H})_{\text {int }}=\left(\frac{1}{12},-\frac{3}{8}\right)$, massless fermions have $\left(\frac{1}{12}, 0\right)$ and massless scalars have $\left(\frac{1}{12}, \frac{1}{8}\right)$. As to the infrared limit of the $\eta$ and $Z_{\psi}$ factors in eq. (22), we know that $\eta(\tau) \approx q^{1 / 24}(1-q+\cdots)$, and we can use formulas (18) to derive $Z_{\Psi}(\bar{\tau}, \mathrm{NS}) \approx$ $\left.\bar{q}^{-1 / 24} \pm 2 \bar{q}^{+11 / 24}\right)$ and $Z_{\Psi}(\bar{\tau}, \mathrm{R}) \approx 2 \bar{q}^{+1 / 12}$. Substituting this asymptotic behavior into eq. (22), we arrive at

$$
\mathcal{B}_{a} \underset{\tau_{2} \rightarrow \infty}{\longrightarrow}-\frac{11}{3} \operatorname{tr}_{V, M=0}\left(Q_{a}^{2}\right)+\frac{2}{3} \operatorname{tr}_{F, M=0}\left(Q_{a}^{2}\right)+\frac{1}{3} \operatorname{tr}_{S, M=0}\left(Q_{a}^{2}\right)=b_{a} \equiv \lim _{t \rightarrow \infty} \mathbf{B}_{a}(t)
$$

(cf. eq. (6)): the string theory indeed yields the same infrared divergence of $\mathcal{W}_{a}$ as the effective low-energy gauge theory. ( Notice that the width of $\Gamma-w_{\Gamma}\left(\tau_{2}\right) \equiv$ 
$\int d \tau_{1} \theta\left(\tau_{1}+i \tau_{2} \in \Gamma\right)$ - is 1 for $\tau_{2} \geq 1$.) Similar to the GUT case, this means that despite the divergence of $\mathcal{W}_{a}^{\text {string }}$, the difference between the bare couplings comes out finite. Let me write down an explicit formula:

$$
\begin{aligned}
\frac{16 \pi^{2}}{g_{a}^{2}}-\frac{16 \pi^{2} k_{a}}{g_{\text {string }}^{2}}= & \int_{\Gamma} \frac{d^{2} \tau}{\tau_{2}}\left(\mathcal{B}_{a}\left(\tau_{1}, \tau_{2}\right)\right. \\
& \left.-b_{a}\right) \\
& +b_{a} \cdot \int_{0}^{\infty} \frac{d \tau_{2}}{\tau_{2}}\left(w_{\Gamma}\left(\tau_{2}\right)-C_{\Lambda}\left(\pi \alpha^{\prime} \tau_{2}\right)^{\star}\right)+k_{a} \cdot 16 \pi^{2} Y .
\end{aligned}
$$

It remains to translate the bare couplings into renormalized couplings evaluated at $\mu=M_{\mathrm{GUT}}$; this is a straightforward exercise for the field-theory coupling $g_{a}$, but how does one chose a renormalization scheme for the string coupling? One logical choice is to use the ultraviolet finiteness of the string theory and to define the renormalized $g_{\text {string }}$ to be the same as the bare $g_{\text {string }}$. However, I prefer a different scheme for the $g_{\mathrm{GUT}}$, namely $g_{\mathrm{GUT}}^{-2} \stackrel{\text { def }}{=}\left(g_{\text {string }}^{\text {bare }}\right)^{-2}+Y$; in this scheme the last term in (24) is precisely canceled, so I do not even have to compute the $Y$ (and I didn't). Of course, I will have to compute the $Y$ if I ever need a value of $g_{\text {string }}^{-2}$ that is accurate to the order $O(1)$, but I do not need it in this article.

Finally, when I translate the bare $g_{a}$ into the $\overline{\mathrm{DR}}$ coupling $g_{a}\left(\mu=M_{\mathrm{GUT}}\right), \mathrm{I}$ add a $b_{a} \cdot\left(\xi-\log \frac{\Lambda^{2}}{M_{\mathrm{GUT}}^{2}}\right)$ term to the left hand side of eq. (24); $\xi$ depends on a choice of the regularization scheme. The second term on the right hand side of (24) can be written in a similar form: $b_{a} \cdot\left(\xi^{\prime}-\log \alpha^{\prime} \Lambda^{2}\right)$; the difference between these two terms is $b_{a} \cdot\left(\xi^{\prime \prime}-\log \alpha^{\prime} M_{\mathrm{GUT}}^{2}\right)$ where $\xi^{\prime \prime}=\xi^{\prime}-\xi$ is a numerical constant. A straightforward exercise in calculus yields $\xi^{\prime \prime}=1+\log (2 / \sqrt{27} \pi)-\gamma \approx-1.6767$ $(\gamma \approx 0.57722$ is the Euler's constant). Now consider the fact that a precise definition of $M_{\mathrm{GUT}}$ in terms of heavy masses is a matter of convention. No such convention has been established for the string-based models, so I consider myself

* To see that $t$ should be identified with $\pi \alpha^{\prime} \tau_{2}$, compare the exponential factors associated with the space-time momentum. For the proper time $t$ this factor is $e^{-t p^{2}}$ (cf. eq. (4)); the corresponding factor for $\tau_{2}$ is $(q \bar{q})^{\alpha^{\prime} p^{2} / 4}=e^{-\pi \tau_{2} \alpha^{\prime} p^{2}}$ (cf. eq. (19)). 
free to define $M_{\mathrm{GUT}} \stackrel{\text { def }}{=} e^{\xi^{\prime \prime} / 2} / \sqrt{\alpha^{\prime}}$; the virtue of this definition is that it reduces the formula for the threshold corrections $\Delta_{a}$ to the first term in eq. (24).

At this point I can write down the main result of this article: one-stringloop threshold corrections to low-energy gauge couplings are related to the model's spectrum via:

$$
\Delta_{a}=\int_{\Gamma} \frac{d^{2} \tau}{\tau_{2}}\left(\mathcal{B}_{a}(\tau, \bar{\tau})-b_{a}\right)
$$

where $b_{a}=\mathcal{B}_{a}(\tau=i \infty)$ and $\mathcal{B}(\tau, \bar{\tau})$ is defined in eq. (22). This result is valid in the $\overline{\mathrm{DR}}$ renormalization scheme for the following definition of the unification scale:

$$
\begin{aligned}
M_{\mathrm{GUT}} \stackrel{\text { def }}{=\frac{2 e^{(1-\gamma) / 2} 3^{-3 / 4}}{\sqrt{2 \pi \alpha^{\prime}}}} & =\frac{e^{(1-\gamma) / 2} 3^{-3 / 4}}{4 \pi} g_{\text {string }} M_{\text {Planck }}^{\star} \\
& \approx g_{\mathrm{GUT}} \cdot 5.27 \cdot 10^{17} \mathrm{GeV} .^{\dagger}
\end{aligned}
$$

I would like to stress that formula (25) is valid for any four-dimensional string model that has no tachyons and is based upon a classical vacuum of the heterotic string, i.e., a world-sheet field theory with exact $(0,1)$ superconformal invariance. ${ }^{[10]}$ No additional requirements are necessary; in particular, a model need not be spacetime supersymmetric.

$\star \mathrm{I}$ am using the following conventions for the couplings: $g_{\mathrm{GUT}}$ and the individual gauge couplings $g_{a}$ are normalized according to the conventions used in the standard model phenomenology and ordinary GUTs. The string coupling $g_{\text {string }}$ is normalized according to the tree-level relation $g_{\text {GUT }} \stackrel{\text { tree }}{=} g_{\text {string. }}$. With this convention, $\alpha^{\prime} M_{\text {Planck }}^{2} g_{\text {string }}^{2}=32 \pi$, which differs by a factor of 2 from a similar formula given in ref. [6].

$\dagger$ The last two digits here should not be taken too seriously since this formula is only accurate in its leading term. 


\section{Threshold Effects in Orbifold Models}

Formulae (25) and (22) express the one-loop threshold corrections in terms of the entire particle spectrum of the model under consideration. Unfortunately, for many string models such as Calabi-Yau compactifications of the ten-dimensional heterotic string $^{[13]}$ only the massless spectra are known. On the other hand, for orbifolds and other models built out of lattices, free fermions, Kac-Moody algebras, minimal Virasoro algebras, etc., writing down the entire spectrum - massless and massive - and computing the traces in formula (22) is a matter of algebra. In particular, for an orbifold with point group $G$, the traces can be expanded as

$$
\operatorname{Tr}_{s_{1}}\left((-)^{s_{2} F} q^{H} \bar{q}^{\bar{H}} \cdot Q_{a}^{2}\right)=\frac{1}{|G|} \sum_{\substack{g, h \in G \\ g h=h g}} \operatorname{Tr}_{\left(g, s_{1}\right)}\left(h \cdot(-)^{s_{2} F} q^{H} \bar{q}^{\bar{H}} \cdot Q_{a}^{2}\right)
$$

and all traces on the right hand side of (27) can be easily written in terms of $\eta$ functions and $\Theta$-functions of various lattices. Actually, the completely untwisted sector $(g=h=1)$ does not contribute anything to the $\mathcal{B}_{a}(\tau, \bar{\tau})$, because this sector by itself is an $N=4$ supersymmetric model. In addition to $\Psi^{\mu}$ it has six more free world-sheet fermions that obey the same boundary conditions while no other world-sheet fields are affected by a choice of a spin structure s. Therefore, the relevant traces over the internal degrees of freedom can be written as $Z_{\Psi}^{3}(\mathbf{s}, \bar{\tau})$ times some trace that does not depend on $\mathbf{s}$. Hence, in the $N=4$ supersymmetric case the right hand side of $(22)$ is proportional to

$$
\sum_{\text {even } \mathbf{s}}(-)^{s_{1}+s_{2}} Z_{\Psi}^{3}(\mathbf{s}, \bar{\tau}) \cdot \frac{d}{d \bar{\tau}} Z_{\Psi}(\mathbf{s}, \bar{\tau})=\frac{1}{4} \frac{d}{d \bar{\tau}} \sum_{\text {even } \mathbf{s}}(-)^{s_{1}+s_{2}} Z_{\Psi}^{4}(\mathbf{s}, \bar{\tau})=0
$$

This means that for $N=4$ supersymmetric models not only the $\beta$-functions vanish, but the one-loop threshold corrections vanish too.

I would like to conclude this article with an example of actually computing the threshold effects for some simple model. Unfortunately, no realistic or almostrealistic models fall into this category, so I shall use an unrealistic example of the 
$Z_{3}$ orbifold. $^{[14]}$ The symmetric $Z_{3}$ orbifold is an $N=1$ supersymmetric model whose low-energy gauge group is $S U(3) \otimes E_{6} \otimes E_{8}$; charged massless matter fields transform as $3(\mathbf{3}, \mathbf{2 7}, \mathbf{1})+27(\mathbf{1}, \mathbf{2 7}, \mathbf{1})+81(\overline{\mathbf{3}}, \mathbf{1}, \mathbf{1})$. The $\beta$-functions are given by $b_{8}=-90, b_{6}=b_{3}=+72$, so the difference between low-energy couplings for the $E_{6}$ and for the $S U(3)$ is due solely to the threshold corrections. Actually, the term 'symmetric $Z_{3}$ orbifold' refers to a nine-parameter family of string models, where the parameters are the radii and the angles of a torus that is modded out by the $Z_{3}$ group. However, out of the nine $(g, h)$ sectors whose contributions total the $\mathcal{B}_{a}$, the only sector affected by the geometry of the torus is the completely untwisted sector $g=h=1$, and that sector is $N=4$ supersymmetric and does not contribute anything at all. Hence, none of the moduli of the $Z_{3}$ orbifold affect the values of the threshold corrections. This feature is probably peculiar to the $Z_{3}$ case: most other orbifolds contain $N=2$ supersymmetric sectors whose contributions do depend on some of the moduli.

As to the actual calculation, it is not too hard to derive algebraic expressions for $\mathcal{B}_{3,6,8}(\tau, \bar{\tau})$ in terms of $\eta(\tau)$ and the $\Theta$-functions of the $S U(3)$ lattice; these expressions are rather long and not particularly interesting. The only interesting feature is that $\mathcal{B}_{3}$ and $\mathcal{B}_{6}$ are exactly equal to each other, so there is no one-loop difference between $g_{3}$ and $g_{6} . \mathcal{B}_{8}$ is different; integrating the difference numerically, I got $\Delta_{3}-\Delta_{8} \approx 11$. When compared to $b_{3}-b_{8}=162$, this threshold correction amounts to a small change in the effective GUT scale (defined as a point at which the renormalization curves for $g_{3}(\mu)$ and $g_{8}(\mu)$ cross each other): it comes out about $3.5 \%$ higher than (26).

Surprisingly, I got exactly the same answer for a different $Z_{3}$ orbifold model in which each of the two $E_{8}$ groups is broken down to an $E_{6} \otimes S U(3)$. For this model $b_{3}-b_{6}=81=162 / 2$, and the difference $\mathcal{B}_{3}-\mathcal{B}_{6}$ is also exactly one half of the expression for $\mathcal{B}_{3}-\mathcal{B}_{8}$ in the first example, so the effective GUT scale is the same in both models. Moreover, if the moduli of the orbifold are chosen such as to yield three extra $S U(3)$ factors in the low-energy gauge group, the running coupling $g_{3}^{\prime}(\mu)$ for those factors crosses both $g_{3}(\mu)$ and $g_{6}(\mu)$ at the same point. It 
is not clear at the moment whether these coincidences are peculiar to $Z_{3}$ orbifolds, or whether they are common to other string models. In the latter case this would be a yet another class of string miracles; further investigation of this subject might be worthwhile.

The author would like to acknowledge many fruitful conversations with T. Banks, M. Peskin, L. Susskind and other members and guests of the SLAC theory group.

\section{REFERENCES}

1. H. Georgi, H. R. Quinn and S. Weinberg, Phys. Rev. Lett. 33 (1974), 451.

2. H. Georgi and S. L. Glashow, Phys. Rev. Lett. 32 (1974), 438

3. S. Weinberg, Phys. Lett. 91B (1980), 51.

4. For review see "Superstring Theory" by M. B. Green, J. H. Schwarz and E. Witten, Cambridge University Press (1987).

5. D. J. Gross, J. A. Harvey, E. Martinec and R. Rohm, Phys. Rev. Lett. 54 (1985), 502, Nucl. Phys. B256 (1985), 253, Nucl. Phys. B267 (1986), 75.

6. P. Ginsparg, Phys. Lett. 197B (1987), 139.

7. V. Kaplunovsky, Phys. Rev. Lett. 55 (1985), 1036.

8. A. Hasenfratz and P. Hasenfratz, Phys. Lett. 93B (1980), 165.

9. J. Polchinski, Comm. Math. Phys. 104 (1986), 37.

10. D. Friedan, E. Martinec and S. Shenker, Nucl. Phys. B271 (1986), 93.

11. C. Callan, I. Klebanov and M. Perry, Nucl. Phys. B278 (1986), 78.

12. A. Sen, Phys. Rev. D32 (1985), 2102.

13. P. Candelas, G. Horowitz, A. Strominger and E. Witten, Nucl. Phys. B258 (1985), 46.

14. L. J. Dixon, J. A. Harvey, C. Vafa and E. Witten, Nucl. Phys. B261 (1985), 671 and B274 (1986), 285. 